American Journal of Agricultural and Biological Sciences 4 (2): 123-130, 2009

ISSN 1557-4989

(C) 2009 Science Publications

\title{
Effects of Converting Secondary Forest on Tropical Peat Soil to Oil Palm Plantation on Carbon Storage
}

\author{
${ }^{1}$ Ch'ng Huck Ywih, ${ }^{1}$ Osumanu Haruna Ahmed, \\ ${ }^{1}$ Nik Muhamad Ab. Majid and ${ }^{2}$ Mohamadu Boyie Jalloh \\ ${ }^{1}$ Department of Crop Science, Faculty of Agriculture and Food Science, \\ University Putra Malaysia, Bintulu Campus, Sarawak, 97008 Bintulu, Sarawak, Malaysia \\ ${ }^{2}$ School of Sustainable Agriculture, University Malaysia Sabah, \\ Locked Bag 2073, 88999 Kota Kinabalu, Sabah, Malaysia
}

\begin{abstract}
Problem statement: Peat has been identified as one of the major groups of soils found in Malaysia. Sarawak as the largest state in Malaysia has the biggest reserve of peat-land. There are about 1.5 million ha of peat-land in Sarawak, which are relatively under developed. As is the case with any plant, oil palm trees do sequester carbon as they grow. Nevertheless, the process of clearing forest in order to establish a plantation may release carbon. Little studies have been done on the comparison of soil organic matter, soil organic carbon and yield of humic acids when secondary forest on peat soil is converted to oil palm plantation. The objective of this study was to compare carbon storage of secondary forest and early stages of oil palm plantations on a tropical peat soil. Approach: Soil samples were collected from the secondary forest, 1, 3, 4 and 5 year old oil palm plantations in Tatau district, Sarawak. Ten samples were taken at random with a peat auger at 0-25 and 25-50 cm depths. The bulk densities at these depths were determined by the coring method. The bulk density method was used to quantify the total carbon, total organic matter, total nitrogen, humic acids and stable carbon at the stated sampling depths on per hectare basis. Results: There were no significant differences in the amounts of stable $\mathrm{C}$ of both secondary forest and different ages of the oil palm plantations at 0-25 and 25-50 cm soil depth. The amounts of stable $C$ in the secondary forest, 1, 3, 4 and 5 year old oil palm plantations at $0-25 \mathrm{~cm}$ depth were generally higher than those in the $25-50 \mathrm{~cm}$ depth. This was attributed to higher yield of HA in the secondary forest, 1, 3, 4 and 5 year old oil palm plantations soil partly due to better humification at the $0-25 \mathrm{~cm}$ soil depth.Conclusion: Conversion of secondary forest on peat to initial stages of oil palm plantation seems to not exert any significant difference on carbon storage in tropical peat soil.
\end{abstract}

Key words: Carbon storage, secondary forest, oil palm plantation, peat, humic acids, soil organic matter, stable carbon

\section{INTRODUCTION}

In the tropics, Soil Organic Matter (SOM) determines the fertility and productivity of soils, especially when soils are highly weathered, with small or no reserves of nutrients and are managed without any external inputs of organic or inorganic fertilizer ${ }^{[1,2]}$. Moreover, at the global scale the type of land use affects the capacity of the soil to act as both a source and a sink of organic matter, nutrients and atmospheric $\mathrm{CO}_{2}$. Soil organic carbon pool contains an estimated $1500 \mathrm{Gt}$ carbon of the total terrestrial carbon store ${ }^{[3]}$.

Tropical rainforest covers about 19.37 million ha of Malaysia's total area and about 8.71 million ha can be found in Sarawak, Malaysia. Land use in Malaysia especially Sarawak has changed significantly because of transmigration and changes in the rural economy. Excessive logging, mining and oil palm cultivation (shifting cultivation) contribute to deforestation in Sarawak.

Peat soils consist of partly decomposed biomass and develop in depressions or wet coastal areas when the rate of biomass production from adapted vegetation (i.e., mangroves, swamp forest) is greater than the rate of decomposition. This is due to the presence of a permanently high water table that prevents aerobic decomposition of plant debris ${ }^{[4]}$. Peat has been identified as one of the major groups of soils found in

Corresponding Author: Osumanu Haruna Ahmed, Department of Crop Science, Faculty of Agriculture and Food Sciences, University Putra Malaysia Bintulu Campus, Sarawak, Malaysia Tel: +6086855406 Fax: +608685415 
Malaysia. Three million ha or $8 \%$ of the area is covered with peat. Sarawak as the largest state in Malaysia has the biggest reserve of peat-land. There are about 1.5 million ha of peat-land in Sarawak, which are relatively under developed. They are located in lowlying coastal depression areas. In their natural state, peat soils have generally been recognized as a problem soil with marginal agricultural capability. Poorly drained and waterlogged for most part of the year. Some of this land is considered suitable for oil palm development due to its rather homogeneous soil features, its constant availability of water and its flatness-all in support of uniform yield characteristics in oil palm ${ }^{[4]}$.

Processes that lead to Soil Organic Carbon (SOC) sequestration are conversion of biomass into humus (including humic acids), aggregation to prevent carbon oxidation and translocation of carbon into sub-soil. It plays an important role in sustaining soil fertility, as it is influenced by land use change, like in the shifting cultivation of oil palm from secondary forest. The clearing and burning of forests can lead to a temporary small increase in SOM as a result of degradation of dead roots $^{[5]}$. However, this organic matter is decomposed rapidly and the net consequence is a loss of organic matter from the soil ${ }^{[6-9]}$. Detwiler ${ }^{[9]}$ estimated that the $\mathrm{C}$ content in tropical forest soils decreased by $40 \%$ where deforestation was followed by arable land use.

As is the case with any plant, oil palm trees do sequester carbon as they grow. Carbon (C) is a basic building block of plant tissue. Nevertheless, the process of clearing forest in order to establish a plantation may release more C. So while a new oil palm plantation may grow faster and sequester $\mathrm{C}$ at a higher annual rate than a naturally regenerating forest, in the end the oil palm plantation may store less carbon (50-90\% less over 20 years) than the original forest cover. The $\mathrm{C}$ losses may be greater when the plantation is established on peatland, which store vast amounts of $\mathrm{C}$ but release it as they are drained.

Forest conversion to agriculture is a typical landuse conversion process elsewhere. Carbon sequestration studies of conversion of secondary forest to oil palm plantation are largely limited. Nonetheless, it is essential to assess the $\mathrm{C}$ pool of present agricultural land-use at sufficiently large scales where there is marked effect of soil, climate and management conditions.

The objective of this study was to compare carbon storage of secondary forest and early stage of oil palm plantations on a tropical peat soil.

\section{MATERIALS AND METHODS}

Systematic sampling method was used in this study to obtain the samples of the peat from secondary forest and oil palm plantations in Tatau district, Sarawak. The size of each experimental plot was $30 \times 40 \mathrm{~m}$. Ten soil samples were taken at random using a peat soil auger at 0-25 and 25-50 cm depth. Each sample was a bulk of three samples. The soils were air dried, pounded and sieved to pass through $2 \mathrm{~mm}$ size.

The bulk densities at these depths were determined by the coring method. The bulk density method was used to quantify Total Carbon (TC), total organic matter, total nitrogen, humic acids and stable carbon at the stated sampling depths on per hectare basis.

The soil $\mathrm{pH}$ was analysed using a glass electrode, SOM, TC and TOC by loss-on ignition method ${ }^{[8,10]}$. Soil total Nitrogen $(\mathrm{N})$ was determined using microKjeldahl method.

The extraction of HA was done using standard procedures but with some modifications. A $5 \mathrm{~g}$ of soil samples were placed in polyethylene centrifuge bottles, $50 \mathrm{~mL}$ of $0.5 \mathrm{M} \mathrm{NaOH}$ solution was added and the bottles were tightly closed with a rubber stopper ${ }^{[11]}$. The samples were equilibrated at room temperature on a reciprocal mechanical shaker at $180 \mathrm{rpm}$ for $24 \mathrm{~h}$. After the extraction period, the side of the bottle was washed using distilled water and the mixture centrifuged at $16,211 \mathrm{G}$ for $15 \mathrm{~min}$. The dark color of the supernatant liquors containing the $\mathrm{HA}$ was decanted and the $\mathrm{pH}$ of the solution was adjusted to 1.0 with $6 \mathrm{M} \mathrm{HCl}$. The HA was allowed to equilibrate at room temperature for $8 \mathrm{~h}$. After $8 \mathrm{~h}$, the supernatant (fulvic acids) was siphoned off from the acidified extract. The remainder of the suspension was transferred to polyethylene bottles and the HA centrifuged. Purification process of HA was done by using the method described by Ahmed et al. ${ }^{[12]}$ with some modifications. The HA was purified by suspending in $50 \mathrm{~mL}$ distilled water and centrifuged at $16,211 \mathrm{G}$ for $10 \mathrm{~min}$ and the supernatant decanted. This procedure was repeated 3 times. The washed HA was oven dried at $40^{\circ} \mathrm{C}$ to a constant weight. The yield of the HA was expressed as percentage of the weight of soil used.

Humification level of HA was determined by using $\mathrm{E}_{4} / \mathrm{E}_{6}$ ratio (465 and $665 \mathrm{~nm}$ ) and the method used for the determination of this ratio was by spectroscopy ${ }^{[13]}$ using a Perkin Elmer Lambda 25 UV/VIS spectrometer.

A $0.003 \mathrm{~g}$ sample of the HA was dissolved in $10 \mathrm{~mL}$ of $0.05 \mathrm{M} \mathrm{NaHCO}_{3}$ for this determination.

The Carboxylic (-COOH), Phenolic (-OH) functional groups and total acidity of $\mathrm{HA}$ were determined by the method described by Inbar et al. ${ }^{[14]}$. A $0.02 \mathrm{~g}$ sample of HA was dissolved in $4 \mathrm{~mL}$ of $0.08 \mathrm{M} \mathrm{NaOH}$ and equilibrated at room temperature on a reciprocal shaker for $30 \mathrm{~min}$. The initial $\mathrm{pH}$ was recorded. The solution was titrated with $0.10 \mathrm{M} \mathrm{HCl}$ to 
pH 2.5 within 15 min. Phenol content was calculated by assuming that $50 \%$ of the phenols were dissociated at $\mathrm{pH}$ 10. Carboxyl content was calculated based on the amount of acid required to titrate the suspension between $\mathrm{pH} 8$ and the end point ( $\mathrm{pH} 2.5)$. Total acidity was calculated by summation of the phenols and carboxyls.

Independent T-test was used to detect significant difference for SOM, TOC, total stable C, HA yield, total $\mathrm{N}$ and $\mathrm{pH}$ of the different ages of oil palm plantations and secondary forest while Tukey's test was used to separate the means of the SOM, TOC, total stable $\mathrm{C}$, HA yield, total $\mathrm{N}$ and $\mathrm{pH}$ between the ages of oil palm plantations and the secondary forest. Statistical Analysis System (SAS) version $9.1^{[15]}$ was used for the statistical analysis.

\section{RESULTS}

The $\mathrm{pH}$ of both secondary forest and oil palm plantations regardless of depth were typical of peat soils ${ }^{[8]}$. There were significant differences between the $\mathrm{pH}(1 \mathrm{M} \mathrm{KCl})$ of the secondary forest and four different ages of oil palm plantations at $0-25$ and $25-50 \mathrm{~cm}$ depths (Table 1). The $\mathrm{pH}$ of water and $1 \mathrm{M} \mathrm{KCl}$ at the depth of 0-25 cm of secondary forest, 1 and 3 year old oil palm plantations were lower than those at the depth of $25-50 \mathrm{~cm}$ except for 4 and 5 year old oil palm plantations which showed opposite effect (Table 2).

The soil bulk densities (Table 3 and 4) at the two depths of both secondary forest and oil palm plantations were found to be within the range reported by Andriesse $^{[4]}$. The bulk densities of 1, 3, 4 and 5 year old oil palm plantations showed no significant difference at 0-25 and 25-50 cm depths except for the secondary forest.

Table 1: $\mathrm{pH}$ of secondary forest and oil palm plantations (different ages)

\begin{tabular}{lll}
\hline Location & $\mathrm{pH}($ water $)$ & $\mathrm{pH}(1 \mathrm{M} \mathrm{KCl})$ \\
\hline (a) Secondary forest & & \\
$0-25 \mathrm{~cm}$ & $3.32 \pm 0.029^{\mathrm{a}}$ & $2.26 \pm 0.021^{\mathrm{a}}$ \\
$25-50 \mathrm{~cm}$ & $3.37 \pm 0.034^{\mathrm{a}}$ & $2.37 \pm 0.025^{\mathrm{b}}$ \\
(b) One year old oil palm plantation & & \\
$0-25 \mathrm{~cm}$ & $3.37 \pm 0.034^{\mathrm{a}}$ & $2.58 \pm 0.029^{\mathrm{a}}$ \\
$25-50 \mathrm{~cm}$ & $3.74 \pm 0.044^{\mathrm{b}}$ & $2.63 \pm 0.028^{\mathrm{b}}$ \\
(c) Three year old oil palm plantation & & \\
$0-25 \mathrm{~cm}$ & $3.31 \pm 0.065^{\mathrm{a}}$ & $2.38 \pm 0.055^{\mathrm{a}}$ \\
$25-50 \mathrm{~cm}$ & $3.63 \pm 0.114^{\mathrm{b}}$ & $2.44 \pm 0.059^{\mathrm{b}}$ \\
(d) Four year old oil palm plantation & & \\
$0-25 \mathrm{~cm}$ & $3.10 \pm 0.073^{\mathrm{a}}$ & $2.45 \pm 0.060^{\mathrm{a}}$ \\
$25-50 \mathrm{~cm}$ & $3.10 \pm 0.049^{\mathrm{a}}$ & $2.34 \pm 0.047^{\mathrm{b}}$ \\
(e) Five year old oil palm plantation & & \\
$0-25 \mathrm{~cm}$ & $3.63 \pm 0.096^{\mathrm{a}}$ & $2.68 \pm 0.131^{\mathrm{a}}$ \\
$25-50 \mathrm{~cm}$ & $3.39 \pm 0.050^{\mathrm{b}}$ & $2.38 \pm 0.036^{\mathrm{b}}$ \\
\hline
\end{tabular}

Note: Means within column with different letters indicate significant difference between soil depths by independent t-test $\mathrm{p} \leq 0.05$
Irrespective of secondary forest, 1, 3 and 5 year old oil palm plantations and soil depths, there were no significant differences in the percentages and quantities of SOM (Table 5 and 6). The percentages and quantities of SOM at $0-25 \mathrm{~cm}$ of 3 and 5 year old oil palm plantations were higher than those at $25-50 \mathrm{~cm}$ depth.

Table 2: Comparison of $\mathrm{pH}$ between secondary forest and oil palm plantations (different ages)

\begin{tabular}{lll}
\hline Location & $\mathrm{pH}$ (water) & $\mathrm{pH}(1 \mathrm{M} \mathrm{KCl})$ \\
\hline (a) 0-25 cm & & \\
Secondary forest & $3.32 \pm 0.029^{\mathrm{bc}}$ & $2.26 \pm 0.025^{\mathrm{b}}$ \\
One year old oil palm plantation & $3.37 \pm 0.034^{\mathrm{ab}}$ & $2.58 \pm 0.029^{\mathrm{ab}}$ \\
Three year old oil palm plantation & $3.31 \pm 0.065^{\mathrm{bc}}$ & $2.38 \pm 0.055^{\mathrm{b}}$ \\
Four year old oil palm plantation & $3.10 \pm 0.073^{\mathrm{c}}$ & $2.45 \pm 0.060^{\mathrm{ab}}$ \\
Five year old oil palm plantation & $3.63^{\mathrm{a}} \pm 0.096^{\mathrm{a}}$ & $2.68 \pm 0.131^{\mathrm{a}}$ \\
(b) 25-50 cm & & \\
Secondary forest & $3.37 \pm 0.034^{\mathrm{b}}$ & $2.37 \pm 0.021^{\mathrm{bc}}$ \\
One year old oil palm plantation & $3.74 \pm 0.044^{\mathrm{a}}$ & $2.63 \pm 0.028^{\mathrm{a}}$ \\
Three year old oil palm plantation & $3.63 \pm 0.114^{\mathrm{ab}}$ & $2.44 \pm 0.059^{\mathrm{b}}$ \\
Four year old oil palm plantation & $3.10 \pm 0.049^{\mathrm{c}}$ & $2.34 \pm 0.047^{\mathrm{c}}$ \\
Five year old oil palm plantation & $3.39 \pm 0.050^{\mathrm{b}}$ & $2.38 \pm 0.036^{\mathrm{bc}}$ \\
\hline
\end{tabular}

Note: Means within column with different letters indicate significant difference between locations by Tukey test at $\mathrm{p} \leq 0.05$

Table 3: Bulk density of secondary forest and oil palm plantations (different ages)

\begin{tabular}{ll}
\hline Location & Bulk density $\left(\mathrm{g} \mathrm{cm}^{-3}\right)$ \\
\hline (a) Secondary forest & $0.299 \pm 0.007^{\mathrm{a}}$ \\
$0-25 \mathrm{~cm}$ & $0.275 \pm 0.004^{\mathrm{b}}$ \\
$25-50 \mathrm{~cm}$ & \\
(b) One year old oil palm plantation & $0.297 \pm 0.004^{\mathrm{a}}$ \\
$0-25 \mathrm{~cm}$ & $0.294 \pm 0.006^{\mathrm{a}}$ \\
$25-50 \mathrm{~cm}$ & \\
(c) Three year old oil palm plantation & $0.299 \pm 0.007^{\mathrm{a}}$ \\
$0-25 \mathrm{~cm}$ & $0.303 \pm 0.006^{\mathrm{a}}$ \\
$25-50 \mathrm{~cm}$ & \\
(d) Four year old oil palm plantation & $0.309 \pm 0.006^{\mathrm{a}}$ \\
$0-25 \mathrm{~cm}$ & $0.293 \pm 0.007^{\mathrm{a}}$ \\
$25-50 \mathrm{~cm}$ & \\
(e) Five year old oil palm plantation & $0.289 \pm 0.002^{\mathrm{a}}$ \\
$0-25 \mathrm{~cm}$ & $0.284 \pm 0.004^{\mathrm{a}}$ \\
$25-50 \mathrm{~cm}$ &
\end{tabular}

Note: Means within column with different letters indicate significant difference between soil depths by independent $t$-test at $\mathrm{p} \leq 0.05$

Table 4: Comparison of bulk density between secondary forest and oil palm plantations (different ages)

\begin{tabular}{ll}
\hline Location & Bulk density $\left(\mathrm{g} \mathrm{cm}^{-3}\right)$ \\
\hline (a) 0-25 cm & $0.299 \pm 0.007^{\mathrm{a}}$ \\
Secondary forest & $0.297 \pm 0.004^{\mathrm{a}}$ \\
One year old oil palm plantation & $0.299 \pm 0.007^{\mathrm{a}}$ \\
Three year old oil palm plantation & $0.309 \pm 0.006^{\mathrm{a}}$ \\
Four year old oil palm plantation & $0.289 \pm 0.002^{\mathrm{a}}$ \\
Five year old oil palm plantation & \\
(b) 25-50 cm & $0.275 \pm 0.004^{\mathrm{b}}$ \\
Secondary forest & $0.294 \pm 0.006^{\mathrm{ab}}$ \\
One year old oil palm plantation & $0.303 \pm 0.006^{\mathrm{a}}$ \\
Three year old oil palm plantation & $0.293 \pm 0.007^{\mathrm{ab}}$ \\
Four year old oil palm plantation & $0.284 \pm 0.004^{\mathrm{ab}}$ \\
\hline Five year old oil palm plantation & Note: Means within column with different letters indicate significant \\
difference between locations by Tukey test at $\mathrm{p} \leq 0.05$
\end{tabular}


Am. J. Agri. \& Biol. Sci., 4 (2): 123-130, 2009

Table 5: Soil organic matter (\%) and corresponding quantities $\left(\mathrm{Mg} \mathrm{ha}^{-1}\right)$ of secondary forest and oil palm plantations (different ages)

\begin{tabular}{|c|c|c|}
\hline Location & $\operatorname{SOM}(\%)$ & $\begin{array}{l}\text { Quantity of SOM } \\
\left(\mathrm{Mg} \mathrm{ha}^{-1}\right)\end{array}$ \\
\hline \multicolumn{3}{|c|}{ (a) Secondary forest } \\
\hline $0-25 \mathrm{~cm}$ & $95.230 \pm 0.535^{\mathrm{a}}$ & $711.840 \pm 4.005^{\mathrm{a}}$ \\
\hline $25-50 \mathrm{~cm}$ & $96.602 \pm 0.325^{\mathrm{b}}$ & $664.080 \pm 2.234^{\mathrm{b}}$ \\
\hline \multicolumn{3}{|c|}{ (b) One year old oil palm plantation } \\
\hline $0-25 \mathrm{~cm}$ & $89.952 \pm 1.357^{\mathrm{a}}$ & $667.890 \pm 3.236^{\mathrm{a}}$ \\
\hline $25-50 \mathrm{~cm}$ & $97.320 \pm 0.342^{b}$ & $740.510 \pm 0.963^{\mathrm{b}}$ \\
\hline \multicolumn{3}{|c|}{ (c) Three year old oil palm plantation } \\
\hline $0-25 \mathrm{~cm}$ & $96.614 \pm 0.420^{\mathrm{a}}$ & $722.190 \pm 1.345^{\mathrm{a}}$ \\
\hline $25-50 \mathrm{~cm}$ & $93.136 \pm 3.947^{\mathrm{b}}$ & $705.500 \pm 5.332^{\mathrm{b}}$ \\
\hline \multicolumn{3}{|c|}{ (d) Four year old oil palm plantation } \\
\hline $0-25 \mathrm{~cm}$ & $85.494 \pm 1.334^{\mathrm{a}}$ & $660.440 \pm 3.383^{\mathrm{a}}$ \\
\hline $25-50 \mathrm{~cm}$ & $92.334 \pm 0.928^{\mathrm{b}}$ & $676.350 \pm 5.974^{\mathrm{b}}$ \\
\hline \multicolumn{3}{|c|}{ (e) Five year old oil palm plantation } \\
\hline $0-25 \mathrm{~cm}$ & $94.332 \pm 1.488^{\mathrm{a}}$ & $680.830 \pm 4.697^{\mathrm{a}}$ \\
\hline $25-50 \mathrm{~cm}$ & $92.850 \pm 1.665^{\mathrm{b}}$ & $659.240 \pm 4.710^{\mathrm{b}}$ \\
\hline
\end{tabular}

Note: Means within column with different letters indicate significant difference between soil depths by independent $t$-test at $\mathrm{p} \leq 0.05$

Table 6: Comparison of soil organic matter (\%) and corresponding quantities $\left(\mathrm{Mg} \mathrm{ha}^{-1}\right)$ between secondary forest and oil palm plantations (different ages)

\begin{tabular}{lll}
\hline & & $\begin{array}{l}\text { Quantity of } \\
\text { LOM }\left(\mathrm{Mg} \mathrm{ha}^{-1}\right)\end{array}$ \\
\hline (a) 0-25 cm & SOM $(\%)$ & \\
Secondary forest & $95.230 \pm 0.535^{\mathrm{a}}$ & $711.840 \pm 4.005^{\mathrm{a}}$ \\
One year old oil palm plantation & $89.952 \pm 1.357^{\mathrm{ab}}$ & $667.890 \pm 3.236^{\mathrm{ab}}$ \\
Three year old oil palm plantation & $96.614 \pm 0.420^{\mathrm{a}}$ & $722.190 \pm 1.345^{\mathrm{a}}$ \\
Four year old oil palm plantation & $85.494 \pm 1.334^{\mathrm{b}}$ & $660.440 \pm 3.383^{\mathrm{b}}$ \\
Five year old oil palm plantation & $94.332 \pm 1.488^{\mathrm{a}}$ & $680.830 \pm 4.697^{\mathrm{a}}$ \\
(b) 25-50 cm & & \\
Secondary forest & $96.602 \pm 0.325^{\mathrm{a}}$ & $664.080 \pm 2.234^{\mathrm{a}}$ \\
One year old oil palm plantation & $97.320 \pm 0.342^{\mathrm{a}}$ & $740.510 \pm 0.963^{\mathrm{a}}$ \\
Three year old oil palm plantation & $93.136 \pm 3.947^{\mathrm{a}}$ & $705.500 \pm 5.332^{\mathrm{a}}$ \\
Four year old oil palm plantation & $92.334 \pm 0.928^{\mathrm{a}}$ & $676.350 \pm 5.974^{\mathrm{a}}$ \\
Five year old oil palm plantation & $92.850 \pm 1.665^{\mathrm{a}}$ & $659.240 \pm 4.710^{\mathrm{a}}$ \\
\hline
\end{tabular}

Note: Means within column with different letters indicate significant difference between locations by Tukey test at $\mathrm{p} \leq 0.05$

On the other hand, the percentages and quantities of SOM of the secondary forest, 1 and 4 year old oil palm plantations at $0-25 \mathrm{~cm}$ were lower than at $25-50 \mathrm{~cm}$ depth. These values were typical of Saprists of Sarawak, Malaysia ${ }^{[4]}$.

There were no significant differences in the percentages and quantities of total $\mathrm{C}$ of secondary forest, 1, 3 and 5 year old oil palm plantations at $0-25$ and $25-50 \mathrm{~cm}$ depth (Table 7). The total $\mathrm{C}$ at $0-25 \mathrm{~cm}$ of 3 and 5 year old oil palm plantations were higher than those of 25-50 cm depth. However, the total $\mathrm{C}$ in secondary forest, 1 and 4 year old oil palm plantations at $0-25 \mathrm{~cm}$ were lower than that of $25-50 \mathrm{~cm}$ depth (Table 8).
Table 7: Comparison of total carbon (\%) and corresponding quantities $\left(\mathrm{Mg} \mathrm{ha}^{-1}\right)$ between secondary forest and oil palm plantations (different ages)

\begin{tabular}{lcl}
\hline Location & Total C (\%) & $\begin{array}{l}\text { Quantity of C } \\
\left(\mathrm{Mg} \mathrm{ha}^{-1}\right)\end{array}$ \\
\hline (a) 0-25 cm & & \\
Secondary forest & $47.615 \pm 0.267^{\mathrm{a}}$ & $355.910 \pm 2.003^{\mathrm{a}}$ \\
One year old oil palm plantation & $44.976 \pm 1.785^{\mathrm{ab}}$ & $333.950 \pm 4.328^{\mathrm{ab}}$ \\
Three year old oil palm plantation & $48.307 \pm 0.210^{\mathrm{a}}$ & $361.090 \pm 1.745^{\mathrm{a}}$ \\
Four year old oil palm plantation & $42.747 \pm 0.667^{\mathrm{b}}$ & $330.220 \pm 5.153^{\mathrm{b}}$ \\
Five year old oil palm plantation & $47.098 \pm 0.769^{\mathrm{a}}$ & $340.280 \pm 3.311^{\mathrm{a}}$ \\
(b) 25-50 cm & & \\
Secondary forest & $48.301 \pm 0.163^{\mathrm{a}}$ & $332.070 \pm 1.120^{\mathrm{a}}$ \\
One year old oil palm plantation & $48.660 \pm 1.714^{\mathrm{a}}$ & $370.250 \pm 0.550^{\mathrm{a}}$ \\
Three year old oil palm plantation & $46.568 \pm 1.973^{\mathrm{a}}$ & $352.750 \pm 2.666^{\mathrm{a}}$ \\
Four year old oil palm plantation & $46.187 \pm 0.470^{\mathrm{a}}$ & $338.320 \pm 3.444^{\mathrm{a}}$ \\
Five year old oil palm plantation & $46.425 \pm 0.832^{\mathrm{a}}$ & $329.620 \pm 5.373^{\mathrm{a}}$ \\
\hline
\end{tabular}

Note: Means within column with different letters indicate significant difference between locations by Tukey test at $\mathrm{p} \leq 0.05$

Table 8: Total carbon (\%) and corresponding quantities $\left(\mathrm{Mg} \mathrm{ha}^{-1}\right)$ of secondary forest and oil palm plantations (different ages)

\begin{tabular}{|c|c|c|}
\hline Location & Total C (\%) & $\begin{array}{l}\text { Quantity of C } \\
\left(\mathrm{Mg} \mathrm{ha}^{-1}\right)\end{array}$ \\
\hline \multicolumn{3}{|c|}{ (a) Secondary forest } \\
\hline $0-25 \mathrm{~cm}$ & $47.615 \pm 0.267^{\mathrm{a}}$ & $355.910 \pm 2.003^{\mathrm{a}}$ \\
\hline $25-50 \mathrm{~cm}$ & $48.301 \pm 0.163^{\mathrm{b}}$ & $332.070 \pm 1.120^{\mathrm{b}}$ \\
\hline \multicolumn{3}{|c|}{ (b) One year old oil palm plantation } \\
\hline $0-25 \mathrm{~cm}$ & 44.976 & $333.950 \pm 4.328^{\mathrm{a}}$ \\
\hline $25-50 \mathrm{~cm}$ & 48.6 & 370.25 \\
\hline \multicolumn{3}{|c|}{ c) Three year old oil palm plantation } \\
\hline $0-25 \mathrm{~cm}$ & 48.307 & 361.09 \\
\hline $25-50 \mathrm{~cm}$ & 46.5 & $352.750 \pm 2.666^{\mathrm{b}}$ \\
\hline \multicolumn{3}{|c|}{ (d) Four year old oil palm plantation } \\
\hline $25 \mathrm{~cm}$ & $42.747 \pm 0.667^{\mathrm{a}}$ & $330.220^{\mathrm{a}} \pm 5.153^{\mathrm{a}}$ \\
\hline $5-50$ & 46.1 & $338.320 \pm 3.444^{\mathrm{b}}$ \\
\hline \multicolumn{3}{|c|}{ (e) Five year old oil $\mathrm{p}$} \\
\hline 25 & 47 & 34 \\
\hline $25-50 \mathrm{~cm}$ & $46.425 \pm 0.832^{\mathrm{b}}$ & $329.620 \pm 5.373^{\mathrm{b}}$ \\
\hline
\end{tabular}

Note: Means within column with different letters indicate significant difference between soil depths by independent $t$-test at $\mathrm{p} \leq 0.05$

Table 9: Total $\mathrm{N}$ and $\mathrm{C} / \mathrm{N}$ ratios of secondary forest and oil palm plantations (different ages)

\begin{tabular}{|c|c|c|}
\hline Location & Total N (\%) & $\mathrm{C} / \mathrm{N}$ ratio \\
\hline \multicolumn{3}{|c|}{ (a) Secondary forest } \\
\hline $0-25 \mathrm{~cm}$ & $2.401 \pm 0.174^{\mathrm{a}}$ & $20.045 \pm 1.565^{\mathrm{a}}$ \\
\hline $25-50 \mathrm{~cm}$ & $2.468 \pm 0.131^{\mathrm{a}}$ & $20.822 \pm 0.989^{\mathrm{a}}$ \\
\hline \multicolumn{3}{|c|}{ (b) One year old oil palm plantation } \\
\hline $0-25 \mathrm{~cm}$ & $2.328 \pm 0.288^{\mathrm{a}}$ & $22.127 \pm 2.929^{\mathrm{a}}$ \\
\hline $25-50 \mathrm{~cm}$ & $1.537 \pm 0.181^{\mathrm{b}}$ & $28.904 \pm 1.912^{b}$ \\
\hline \multicolumn{3}{|c|}{ (c) Three year old oil palm plantation } \\
\hline $0-25 \mathrm{~cm}$ & $1.304 \pm 0.092^{\mathrm{a}}$ & $38.641^{\mathrm{a}} \pm 2.568^{\mathrm{a}}$ \\
\hline $25-50 \mathrm{~cm}$ & $0.966 \pm 0.111^{\mathrm{b}}$ & $43.422^{\mathrm{b}} \pm 2.653^{\mathrm{b}}$ \\
\hline \multicolumn{3}{|c|}{ (d) Four year old oil palm plantation } \\
\hline $0-25 \mathrm{~cm}$ & 1.375 & $32.968 \pm 2.533^{\mathrm{a}}$ \\
\hline $25-50$ & $049^{\mathrm{a}}$ & $39.175 \pm 1.317^{\mathrm{b}}$ \\
\hline \multicolumn{3}{|c|}{ (e) Five year old oil palm plantation } \\
\hline $0-25 \mathrm{~cm}$ & $1.369 \pm 0.059^{\mathrm{a}}$ & $34.841 \pm 1.202^{\mathrm{a}}$ \\
\hline $25-50 \mathrm{~cm}$ & $1.120 \pm 0.139^{\mathrm{a}}$ & $39.993 \pm 2.394^{\mathrm{b}}$ \\
\hline
\end{tabular}

Note: Means within column with different letters indicate significant difference between soil depths by independent t-test at $p \leq 0.05$

The soil total $\mathrm{N}$ of 1 and 3 year old oil palm plantations significantly decreased down the soil profile (Table 9) On the other hand, there were no significant differences in the total $\mathrm{N}$ between the depths of $0-25$ 
and 25-50 cm of secondary forest, 4 and 5 years old oil palm plantations. The percentages of $\mathrm{N}$ obtained for the different ages of oil palm plantations were in the range reported elsewhere. There was significant difference in the $\mathrm{C} / \mathrm{N}$ ratios of the secondary forest and different ages of oil palm plantations at the depths of 0-25 and 25$50 \mathrm{~cm}$ (Table 10).

The percentages of $\mathrm{HA}$ yields and the corresponding quantities in $\mathrm{Mg} \mathrm{ha}^{-1}$ of the secondary forest at $0-25$ and $25-50 \mathrm{~cm}$ depths were not statistically different. Similar observation was made for the different ages of the oil palm plantations (Table 11). However, the percentage yield of HA and the quantity of $\mathrm{HA}$ in $\mathrm{Mg} \mathrm{ha}^{-1}$ at $0-25$ and 25-50 cm depth of the 3, 4 and 5 year old oil palm plantation were significantly greater than those of secondary forest and the 1 year old oil palm plantation (Table 12).

There were no significant differences in the quantities of stable $\mathrm{C}$ of both secondary forest and different ages of oil palm plantations at 0-25 and 25$50 \mathrm{~cm}$ (Table 13). The quantities of stable $\mathrm{C}$ of the

Table 10: Comparison of total $\mathrm{N}$ and $\mathrm{C} / \mathrm{N}$ ratios between secondary forest and oil palm plantations (different ages)

\begin{tabular}{lll}
\hline Location & Total N $(\%)$ & C/N ratio \\
\hline (a) 0-25 cm & & \\
Secondary forest & $2.401 \pm 0.174^{\mathrm{a}}$ & $20.045 \pm 1.565^{\mathrm{b}}$ \\
One year old oil palm plantation & $2.328 \pm 0.288^{\mathrm{a}}$ & $22.127 \pm 2.929^{\mathrm{b}}$ \\
Three year old oil palm plantation & $1.304 \pm 0.092^{\mathrm{a}}$ & $38.641 \pm 2.568^{\mathrm{a}}$ \\
Four year old oil palm plantation & $1.375 \pm 0.116^{\mathrm{a}}$ & $32.968 \pm 2.533^{\mathrm{a}}$ \\
Five year old oil palm plantation & $1.369 \pm 0.059^{\mathrm{a}}$ & $34.841 \pm 1.202^{\mathrm{a}}$ \\
(b) 25-50 cm & & \\
Secondary forest & $2.468 \pm 0.131^{\mathrm{a}}$ & $20.822 \pm 0.989^{\mathrm{b}}$ \\
One year old oil palm plantation & $1.537 \pm 0.181^{\mathrm{b}}$ & $28.904 \pm 1.912^{\mathrm{b}}$ \\
Three year old oil palm plantation & $0.966 \pm 0.111^{\mathrm{b}}$ & $43.422 \pm 2.653^{\mathrm{a}}$ \\
Four year old oil palm plantation & $1.193 \pm 0.049^{\mathrm{a}}$ & $39.175 \pm 1.317^{\mathrm{ab}}$ \\
Five year old oil palm plantation & $1.120 \pm 0.139^{\mathrm{a}}$ & $39.993 \pm 2.394^{\mathrm{ab}}$ \\
\hline
\end{tabular}

Note: Means within column with different letters indicate significant difference between locations by Tukey test at $\mathrm{p} \leq 0.05$

Table 11: Humic acids yield (\%) and corresponding quantities $\left(\mathrm{Mg} \mathrm{ha}^{-1}\right)$ in secondary forest and oil palm plantations (different ages)

\begin{tabular}{|c|c|c|}
\hline Location & HA yield $(\%)$ & $\begin{array}{l}\text { Quantity of } \\
\text { HA }\left(\mathrm{Mg} \mathrm{ha}^{-1}\right)\end{array}$ \\
\hline \multicolumn{3}{|c|}{ (a) Secondary forest } \\
\hline $0-25 \mathrm{~cm}$ & $38.032 \pm 0.926^{\mathrm{a}}$ & $284.290 \pm 0.934^{\mathrm{a}}$ \\
\hline $25-50 \mathrm{~cm}$ & $38.410 \pm 0.907^{\mathrm{a}}$ & $264.270 \pm 4.012^{\mathrm{a}}$ \\
\hline \multicolumn{3}{|c|}{ (b) One year old oil palm plantation } \\
\hline $0-25 \mathrm{~cm}$ & $38.862 \pm 1.686^{\mathrm{a}}$ & $288.550 \pm 4.346^{\mathrm{a}}$ \\
\hline $25-50 \mathrm{~cm}$ & $33.640 \pm 2.555^{\mathrm{a}}$ & $247.250 \pm 4.410^{\mathrm{a}}$ \\
\hline \multicolumn{3}{|c|}{ (c) Three year old oil palm plantation } \\
\hline $0-25 \mathrm{~cm}$ & $43.744 \pm 0.770^{\mathrm{a}}$ & $326.990 \pm 1.308^{\mathrm{a}}$ \\
\hline $25-50 \mathrm{~cm}$ & $39.074 \pm 1.882^{\mathrm{a}}$ & $295.990 \pm 1.628^{\mathrm{a}}$ \\
\hline \multicolumn{3}{|c|}{ (d) Four year old oil palm plantation } \\
\hline $0-25 \mathrm{~cm}$ & $40.312 \pm 0.667^{\mathrm{a}}$ & $311.410 \pm 2.623^{\mathrm{a}}$ \\
\hline $25-50 \mathrm{~cm}$ & $42.170 \pm 0.928^{\mathrm{a}}$ & $308.900 \pm 2.897^{\mathrm{a}}$ \\
\hline \multicolumn{3}{|c|}{ (e) Five year old oil palm plantation } \\
\hline $0-25 \mathrm{~cm}$ & $40.520 \pm 0.649^{\mathrm{a}}$ & $292.720 \pm 4.689^{\mathrm{a}}$ \\
\hline $25-50 \mathrm{~cm}$ & $41.180 \pm 0.498^{\mathrm{a}}$ & $292.380 \pm 3.540^{\mathrm{a}}$ \\
\hline
\end{tabular}

Note: Means within column with different letters indicate significant difference between soil depths by independent t-test at $\mathrm{p} \leq 0.05$

secondary forest, $1,3,4$ and 5 year old oil palm plantations at the depth of $0-25 \mathrm{~cm}$ were generally higher than those in the $25-50 \mathrm{~cm}$ although there was no significant difference between the depths (Table 14).

Table 12: Comparison of humic acids yield (\%) and corresponding quantities $\left(\mathrm{Mg} \mathrm{ha}^{-1}\right)$ between secondary forest and oil palm plantations (different ages)

\begin{tabular}{lll}
\hline Location & HA yield (\%) & $\begin{array}{l}\text { Quantity of } \\
\text { HA }\left(\mathrm{Mg} \mathrm{ha}^{-1}\right)\end{array}$ \\
\hline (a) 0-25 cm & & \\
Secondary forest & $38.032 \pm 0.926^{\mathrm{b}}$ & $284.290 \pm 0.934^{\mathrm{b}}$ \\
One year old oil palm plantation & $38.862 \pm 1.686^{\mathrm{b}}$ & $288.550 \pm 4.346^{\mathrm{b}}$ \\
Three year old oil palm plantation & $43.744 \pm 0.770^{\mathrm{a}}$ & $326.990 \pm 1.308^{\mathrm{a}}$ \\
Four year old oil palm plantation & $40.312 \pm 0.667^{\mathrm{ab}}$ & $311.410 \pm 2.623^{\mathrm{ab}}$ \\
Five year old oil palm plantation & $40.520 \pm 0.649^{\mathrm{ab}}$ & $292.720 \pm 4.689^{\mathrm{ab}}$ \\
(b) 25-50 cm & & \\
Secondary forest & $38.410 \pm 0.907^{\mathrm{b}}$ & $264.270 \pm 4.012^{\mathrm{b}}$ \\
One year old oil palm plantation & $33.640 \pm 2.555^{\mathrm{b}}$ & $247.250 \pm 4.410^{\mathrm{b}}$ \\
Three year old oil palm plantation & $39.074 \pm 1.882^{\mathrm{ab}}$ & $295.990 \pm 1.628^{\mathrm{ab}}$ \\
Four year old oil palm plantation & $42.170 \pm 0.928^{\mathrm{a}}$ & $308.900 \pm 2.897^{\mathrm{a}}$ \\
Five year old oil palm plantation & $41.180 \pm 0.498^{\mathrm{ab}}$ & $292.380 \pm 3.540^{\mathrm{ab}}$ \\
\hline No Means &
\end{tabular}

Note: Means within column with different letters indicate significant difference between locations by Tukey test at $\mathrm{p} \leq 0.05$

Table 13: Carbon in HA (\%) and quantity of stable $\mathrm{C}\left(\mathrm{Mg} \mathrm{ha}^{-1}\right)$ in secondary forest and oil palm plantations (different ages)

\begin{tabular}{|c|c|c|}
\hline Location & $\begin{array}{l}\text { Carbon in } \\
\text { HA }(\%)\end{array}$ & $\begin{array}{l}\text { Stable C in HA } \\
\left(\mathrm{Mg} \mathrm{ha}^{-1}\right)\end{array}$ \\
\hline \multicolumn{3}{|c|}{ (a) Secondary forest } \\
\hline $0-25 \mathrm{~cm}$ & $47.626 \pm 0.573^{\mathrm{a}}$ & $135.430 \pm 3.970^{\mathrm{a}}$ \\
\hline $25-50 \mathrm{~cm}$ & $48.452 \pm 1.574^{\mathrm{a}}$ & $127.810 \pm 4.211^{\mathrm{a}}$ \\
\hline \multicolumn{3}{|c|}{ (b) One year old oil palm plantation } \\
\hline $0-25 \mathrm{~cm}$ & $47.612 \pm 0.999^{\mathrm{a}}$ & $137.670 \pm 4.419^{\mathrm{a}}$ \\
\hline $25-50 \mathrm{~cm}$ & $47.662 \pm 0.686^{\mathrm{a}}$ & $117.410 \pm 1.556^{\mathrm{a}}$ \\
\hline \multicolumn{3}{|c|}{ (c) Three year old oil palm plantation } \\
\hline $0-25 \mathrm{~cm}$ & $47.216 \pm 0.210^{\mathrm{a}}$ & $154.390 \pm 1.615^{\mathrm{a}}$ \\
\hline $25-50 \mathrm{~cm}$ & $46.920 \pm 0.329^{\mathrm{a}}$ & $138.710 \pm 5.000^{\mathrm{a}}$ \\
\hline \multicolumn{3}{|c|}{ (d) Four year old oil palm plantation } \\
\hline $0-25 \mathrm{~cm}$ & $47.964 \pm 0.523^{\mathrm{a}}$ & $149.420 \pm 1.231^{\mathrm{a}}$ \\
\hline $25-50 \mathrm{~cm}$ & $46.188 \pm 3.420^{\mathrm{a}}$ & $142.290 \pm 3.101^{\mathrm{a}}$ \\
\hline \multicolumn{3}{|c|}{ (e) Five year old oil palm plantation } \\
\hline $0-25 \mathrm{~cm}$ & $48.380 \pm 0.950^{\mathrm{a}}$ & $141.760 \pm 4.638^{\mathrm{a}}$ \\
\hline $25-50 \mathrm{~cm}$ & $47.718 \pm 0.633^{\mathrm{a}}$ & $139.530 \pm 2.668$ \\
\hline
\end{tabular}

Note: Means within column with different letters indicate significant difference between soil depths by independent $t$-test at $\mathrm{p} \leq 0.05$.

Table 14: Comparison of carbon in HA (\%) and quantity of stable C $\left(\mathrm{Mg} \mathrm{ha}{ }^{-1}\right)$ between secondary forest and oil palm plantations (different ages)

\begin{tabular}{lll}
\hline Location & $\begin{array}{l}\text { Carbon in } \\
\text { HA (\%) }\end{array}$ & $\begin{array}{l}\text { Stable C in HA } \\
\left(\mathrm{Mg} \mathrm{ha}^{-1}\right)\end{array}$ \\
\hline (a) $\mathbf{0 - 2 5} \mathbf{~ c m}$ & &
\end{tabular}

\section{(a) $0-25 \mathrm{~cm}$}

Secondary forest

One year old oil palm plantation

Three year old oil palm plantation

Four year old oil palm plantation

Five year old oil palm plantation

(b) $25-50 \mathrm{~cm}$

Secondary forest

One year old oil palm plantation $\quad 47.662 \pm 0.686^{\mathrm{a}} \quad 117.410 \pm 1.556^{\mathrm{a}}$

Three year old oil palm plantation $\quad 46.920 \pm 0.329^{\mathrm{a}} \quad 138.710 \pm 5.000^{\mathrm{a}}$

Four year old oil palm plantation $\quad 46.188 \pm 3.420^{\mathrm{a}} \quad 142.290 \pm 3.101^{\mathrm{a}}$

Five year old oil palm plantation $\quad 47.718 \pm 0.633^{\mathrm{a}} \quad 139.530 \pm 2.668^{\mathrm{a}}$

Note: Means within column with different letters indicate significant difference between locations by Tukey test at $\mathrm{p} \leq 0.05$. 


\section{DISCUSSION}

The significantly higher $\mathrm{pH}(1 \mathrm{M} \mathrm{KCl})$ values at $25-50 \mathrm{~cm}$ of the secondary forest and the 1 and 3 year old oil palm plantations compared to those at $0-25 \mathrm{~cm}$ soil depth (Table 1) could be attributed to the leaching of basic cations from $0-25$ to $25-50 \mathrm{~cm}$. However, no such observation was made for $\mathrm{pH}$ (water) whereby the $\mathrm{pH}$ ranged from 3.10-3.74 and were in the range reported by Murtedza et al. ${ }^{[16]}$. This may be because the $\mathrm{KCl}$ used was more effective in displacing the hydrogen ions. The significant differences between the soil $\mathrm{pH}$ of the secondary forest and the different ages of oil palm plantations regardless of soil depths suggest that different soil management has significant effect on the soil $\mathrm{pH}$. The variations within this range of $\mathrm{pH}$ were due to specific locations of peat swamp ${ }^{[4]}$. According to Andriesse ${ }^{[8]}$, these variations occur in different sections of the peat where the surface layer of the thickest section are lower in $\mathrm{pH}$ compared to the shallow organic soils near the edge.

The values of the bulk density of the secondary forest and different ages of oil palm plantations were below $0.5 \mathrm{~g} \mathrm{~cm}^{-3}$ (Table 3) suggesting that the peats were well decomposed sapric materials ${ }^{[8]}$. The general absence of significant difference between the bulk densities of the $1,3,4$ and 5 year old oil palm plantations regardless of depth was because before planting, the soil is usually compacted using machinery. The soil bulk density of the secondary forest was significantly higher at $0-25$ than $25-50 \mathrm{~cm}$ depth probably because of machinery and other traffic. The absence of significant difference in the soil bulk densities of the different ages of oil palm plantation irrespective of depth could be partly associated with no significant difference in SOM (Table 3 and 4).

Irrespective of secondary forest, 1, 3 and 5 year old oil palm plantations and soil depth, there were no significant differences in the percentages and quantities of SOM within the same depth (Table 5 and 6). This suggests that SOM in the secondary forest, 1, 3 and 5 year old of oil palm plantations have reached equilibrium. The variations of the amount of SOM between the two different depths of the different ages of oil palm plantation could be due to mixing and compaction process usually carried out by the management of oil palm plantation during forest clearance for planting of the oil palm plants. This may have led to the uneven decomposition rate of organic materials between the two depths.

There were no significant differences in the percentage and quantities of total $\mathrm{C}$ of secondary forest
1,3 and 5 year old oil palm plantations within $0-25$ and $25-50 \mathrm{~cm}$ depths (Table 7 and 8). This observation could be ascribed to the absence of significant differences in the percentage and quantities of SOM within the $0-25$ and $25-50 \mathrm{~cm}$ depths of the forest, 1,3 and 5 year old oil palm plantations soils. This finding is partly consistent with the observation that SOM is a major source and sink of atmospheric $\mathrm{C}$ in the global $\mathrm{C}$ cycle $^{[17]}$. The TC in the secondary forest, 3 and 5 years old oil palm plantations at of $0-25 \mathrm{~cm}$ depth was higher than at $25-50 \mathrm{~cm}$ depth.

This pattern is associated with deep organic soils due to large content of ligneous materials in oligotrophic Histosols ${ }^{[16]}$. However, the quantity of TC in 25-50 cm depth of the 1 and 4 year old oil palm plantations was higher than at $0-25 \mathrm{~cm}$ depth.

The soil total $\mathrm{N}$ of the 1 and 3 year old oil palm plantations significantly decreased down the soil profile. On the other hand, there was no significant difference in the total $\mathrm{N}$ between the $0-25$ and $25-50 \mathrm{~cm}$ depths (secondary forest, 4 and 5 year old oil palm plantations). However, the soil total $\mathrm{N}$ of all the different ages of oil palm plantations at the $25-50 \mathrm{~cm}$ depth was generally lower than at $0-25 \mathrm{~cm}$ depth. This observation was consistent with the general observation that soil $\mathrm{N}$ decreases with decreasing soil depth because of decrease in organic $\mathrm{N}$. The soil total $\mathrm{N}$ of the secondary forest at $25-50 \mathrm{~cm}$ depth was higher than at $0-25 \mathrm{~cm}$ depth which could be due to the leaching of $\mathrm{N}$ from $0-25 \mathrm{~cm}$ and accumulation in $25-50 \mathrm{~cm}$ depth (Tables 9 and 10).

The increase in $\mathrm{C} / \mathrm{N}$ ratio with increasing soil depth in the secondary forest and different ages of oil palm plantations suggests that there was more humification at $0-25 \mathrm{~cm}$ than in $25-50 \mathrm{~cm}$ depth. The lower $\mathrm{C} / \mathrm{N}$ ratio of the secondary forest compared to the different ages of the oil palm plantation could be due to the significant accumulation of $\mathrm{N}$ at $25-50 \mathrm{~cm}$ depth as discussed previously.

The percentages of HA yield and corresponding quantities in $\mathrm{Mg} \mathrm{ha}^{-1}$ of the secondary forest at 0-25 and $25-50 \mathrm{~cm}$ depths were not statistically different. Similar observations were made for the different ages of oil palm plantations (Table 11). However, the percentages of yield $\mathrm{HA}$ and the quantity of $\mathrm{HA}$ in $\mathrm{Mg} \mathrm{ha}^{-1}$ at $0-25$ and $25-50 \mathrm{~cm}$ of 3,4 and 5 year old oil palm plantations were significantly greater than those of secondary forest and the 1 year old oil palm plantation (Table 12). This finding was probably because of low $\mathrm{N}$ for efficient conversion of biomass $\mathrm{C}$ into humus $\mathrm{C}$ in the secondary forest and the 1 year old oil palm plantation, a process required for humification of biomass. 
Am. J. Agri. \& Biol. Sci., 4 (2): 123-130, 2009

Table 15: Comparison of ranges of phenolic-OH, carboxylic, total acidity and $\mathrm{E}_{4} / \mathrm{E}_{6}$ ratio of HA of secondary forest and different ages of oil palm plantations with related reports

\begin{tabular}{|c|c|c|c|c|c|c|c|c|}
\hline \multirow[b]{2}{*}{ Location } & \multirow[b]{2}{*}{$\mathrm{E}_{4} / \mathrm{E}_{6}$ ratios } & \multicolumn{6}{|l|}{$\mathrm{cmol} \mathrm{kg}^{-1}$} & \multirow[b]{2}{*}{ Range } \\
\hline & & $\begin{array}{l}\text { Carboxylic } \\
\text { Range }\end{array}$ & $-\mathrm{COOH}$ & $\begin{array}{l}\text { Phenolic } \\
\text { Range }\end{array}$ & $-\mathrm{OH}$ & $\begin{array}{l}\text { Total } \\
\text { Range }\end{array}$ & acidity & \\
\hline \multicolumn{9}{|c|}{ Secondary forest: } \\
\hline $0-25 \mathrm{~cm}$ & 7.850 & $6-8$ & 500 & $240-540$ & 400 & $150-440$ & 900 & $390-980$ \\
\hline $25-50 \mathrm{~cm}$ & 8.144 & & 550 & & 400 & & 950 & \\
\hline \multicolumn{9}{|c|}{ One year old oil palm plantation: } \\
\hline $0-25 \mathrm{~cm}$ & 6.618 & & 510 & & 360 & & 870 & \\
\hline $25-50 \mathrm{~cm}$ & 7.150 & & 450 & & 360 & & 810 & \\
\hline \multicolumn{9}{|c|}{ Three year old oil palm plantation: } \\
\hline $0-25 \mathrm{~cm}$ & 7.042 & & 530 & & 420 & & 950 & \\
\hline $25-50 \mathrm{~cm}$ & 6.985 & & 550 & & 380 & & 930 & \\
\hline \multicolumn{9}{|c|}{ Four year old oil palm plantation: } \\
\hline $0-25 \mathrm{~cm}$ & 7.509 & & 530 & & 400 & & 930 & \\
\hline $25-50 \mathrm{~cm}$ & 7.588 & & 560 & & 400 & & 960 & \\
\hline \multicolumn{9}{|c|}{ Five year old oil palm plantation: } \\
\hline $0-25 \mathrm{~cm}$ & 7.621 & & 600 & & 400 & & 1000 & \\
\hline $25-50 \mathrm{~cm}$ & 7.154 & & 490 & & 400 & & 890 & \\
\hline
\end{tabular}

$\operatorname{Tan}^{[18]}$, Schnitzer $^{[19]}$

There was no significant difference in the quantity of stable $\mathrm{C}$ for both the secondary forest and different ages of oil palm plantation at $0-25$ and $25-50 \mathrm{~cm}$ soil depth (Table 13 and 14). This shows that conversion of secondary forest to oil palm plantations at initial stages (till 5 years old) does not exert any difference in the amount of $\mathrm{C}$ sequestered in the peat soil. Since the $\mathrm{C}$ in $\mathrm{HA}$ is more stable ${ }^{[20]}$, it is more realistic to quantify the amount of $\mathrm{C}$ sequestered upon the conversion of secondary forest on peat to oil palm plantations at initial stages.

The relatively high $\mathrm{E}_{4} / \mathrm{E}_{6}$ values in the secondary forest and different ages of oil palm plantations indicate prominence of aliphatic components or the HA in this study were of low molecular weights ${ }^{[18,21]}$. The effectiveness of washing the HA with distilled water is to indicate its purity without altering its chemical characteristics. The total acidity, carboxylic$\mathrm{COOH}$ and phenolic-OH of the secondary forest and different ages of oil palm plantations (Table 15) were found to be consistent with the ranges reported by other researchers ${ }^{[21]}$.

\section{CONCLUSION}

Conversion of secondary forest on peat to initial stages of oil palm plantation seems to not exert any significant difference on carbon storage in tropical peat soil.

\section{ACKNOWLEDGEMENT}

The authors acknowledge the financial support (Fundamental Research Scheme) of this research received from the Ministry of Higher Education, Malaysia via University Putra Malaysia.

\section{REFERENCES}

1. Feller, C. and M.H. Beare, 1997. Physical control of soil organic matter dynamics in the tropics. Geoderma, 79: 69-116. DOI: 10.1016/S00167061(97)00039-6

2. Zech, W., N. Senesi, G. Guggenberger, K. Kaiser, J. Lehmann and T.M. Miano et al., 1997. Factors controlling humification and mineralization of soil organic matter I the tropics. Geoderma, 79: 117-161. DOI: 10.1016/S0016-7061(97)00040-2

3. Amundson, R., 2001. The carbon budget in soils. Ann. Rev. Earth Planet Sci., 29: 535-562. DOI: 10.1146/annurev.earth.29.1.535

4. Andriesse, J.P., 1988. Nature and management of tropical peat soils. FAO. Soils Bull., 59: 165.

5. Nye, P.H. and D.J. Greenland, 1964. Changes in the soil after clearing tropical forest. Plant Soil, 21: 101-112. DOI: 10.1007/BF01373877

6. Schimel, D.S., D.C. Coleman and K.A. Horton, 1985. Soil organic matter dynamics in paired rangeland and cropland toposequences in North Dakota. Geoderma, 36: 201-214. DOI: 10.1016/0016-7061(85)90002-3

7. Ellert, B.H. and E.G. Gregorich, 1996. Storage of carbon, nitrogen and phosphorus in cultivated and adjacent forested soils of Ontario. Soil Sci., 161: 587-603.

http://journals.lww.com/soilsci/toc/1996/09000 
8. Shang, C. and H. Tiessen, 1997. Organic matter lability in a tropical oxisol: Evidence from shifting cultivation, chemical oxidation, particle size, density and magnetic fractionations. Soil Sci., 162: 795-807. http://journals.lww.com/soilsci/toc/1997/11000

9. Detwiler, R.P., 1986. Land use change and the global carbon cycle: The role of tropical soils. Biogeochemistry, 2: 67-93. DOI: 10.1007/BF02186966

10. Cheftetz, B., P.H. Hatcher, Y. Hadar and Y. Chen, 1996. Chemical and biological characterization of organic matter during composting of municipal solid waste. J. Environ. Q., 25: 776-785. http://jeq.scijournals.org/cgi/reprint/25/4/776

11. Gracia, D., J. Cegarra, M.P. Bernal, A. Navarro, 1993. Comparative evaluation of methods employing alkali and sodium pyrophosphate to extract humic substances from peat. Commun. Soil Sci. Plant Anal., 24: 1481-1494. DOI: 10.1080/00103629309368893

12. Ahmed, O.H., M.H.A. Husni, A.R. Anuar, M.M. Hanafi and E.D.S. Angela, 2004. A modified way of producing humic acid from composted pineapple leaves. J. Sustainable Agric., 25: 129-139. DOI: 10.1300/J064v25n01_10

13. Stevenson, F.J., 1994. Humus Chemistry: Genesis, Composition and Reactions. 2nd Edn., John Wiley and Sons, New York, ISBN: 0471594741.

14. Inbar, Y., Y. Chen and Y. Hadar, 1990. Humic substances formed during the composting of organic matter. Soil Sci. Soc. Am. J., 54: 1316-1323. http://soil.scijournals.org/cgi/reprint/54/5/1316
15. SAS., 2001. SAS/STATS Software. SAS Institute, Cary, NC., ISBN: 1580258506.

16. Murtedza, M., E. Padmanabhan, B.L.H. Mei and W.B. Siong, 2000. Status Report-The Peat Soils of Sarawak. Kuching: University Malaysia Sarawak. http://www.strapeat.alterra.nl/download/12\%20pe at\%20soils\%20of\%20Sarawak.pdf

17. Brady, N.C. and R.R. Weil, 2002. The Nature and Properties of Soils. 13th Edn., Prentice Hall, New Jersey, ISBN: 0130167630.

18. Tan, K.H., 2003. Humic Matter in Soil and the Environment: Principles and Controversies. 1st Edn., Marcel Dekker Inc. New York, ISBN: 0824742729.

19. Schnitzer, M. and C.M. Preston, 1986. Analysis of humic acids by solution and solid-state carbon-13 nuclear magnetic resonance. Soil Sci. Soc. Am. J., 50: 326-331.

http://soil.scijournals.org/cgi/reprint/50/2/326

20. Milori, D.M.B.P., L. Martin-Neto, C. Bayer, J. Mielniczuk and V.S. Bagnato, 2002. Humification degree of soil humic acids determined by fluorescence spectroscopy. Soil Sci., 167: 739-749. http://journals.lww.com/soilsci/toc/2002/11000

21. Fong, S.S. and M. Mohamed, 2007. Chemical characterization of humic substances occurring in the peats of Sarawak, Malaysia. Organ. Geochem., 38: $967-976$.

DOI: 10.1016/j.orggeochem.2006.12.010 